\title{
Stealth configurations in vector-tensor theories of gravity
}

\author{
Javier Chagoya, Gianmassimo Tasinato \\ Department of Physics, Swansea University, Swansea, SA2 8PP, U.K.
}

\begin{abstract}
Studying the physics of compact objects in modified theories of gravity is important for understanding how future observations can test alternatives to General Relativity. We consider a subset of vector-tensor Galileon theories of gravity characterized by new symmetries, which can prevent the propagation of the vector longitudinal polarization, even in absence of Abelian gauge invariance. We investigate new spherically symmetric and slowly rotating solutions for these systems, including an arbitrary matter Lagrangian. We show that, under certain conditions, there always exist stealth configurations whose geometry coincides with solutions of Einstein gravity coupled with the additional matter. Such solutions have a non-trivial profile for the vector field, characterized by independent integration constants, which extends to asymptotic infinity. We interpret our findings in terms of the symmetries and features of the original vector-tensor action, and on the number of degrees of freedom that it propagates. These results are important to eventually describe gravitationally bound configurations in modified theories of gravity, such as black holes and neutron stars, including realistic matter fields forming or surrounding the object.
\end{abstract}

\section{Introduction}

The physics of black holes and other gravitationally bound objects can probe non-perturbative aspects of gravitational theories, in regimes that deviate from weak-field approximations. This is particularly important to explore modified gravity theories equipped with screening mechanisms, motivated by the dark energy problem, which satisfy the strong constraints on deviations from General Relativity (GR) in the weak-field limit. See [1] for a review. The existence of non-trivial black hole solutions in scalar and vector-tensor theories of gravity is challenged by powerful no-hair theorems, which can forbid the existence of solutions with scalar or vector hairs (see e.g. the nice review [2]). Ways out to these negative results exist, avoiding explicit or implicit assumptions at the base of the no-hair theorems. For example, in shift symmetric Horndeski scalar-tensor theories [3], a theorem by Hui and Nicolis generally forbids the existence of static black hole configurations with non-trivial scalar hair [4]. Sotiriou and Zhou [5,6] found a way to avoid one of the hypothesis of the theorem, by selecting particular, non-analytic forms for the free functions characterizing Horndeski theories. Many generalizations of these results have then been developed, see e.g. [7-9] for comprehensive reviews.

In this work, we investigate spherically symmetric and slowly rotating configurations in the vectortensor Galileon theories of gravity [10-12] introduced to address the dark energy problem. In this case, Bekenstein classic no-hair theorems [13] can be circumvented, leading to more general families of black hole solutions, first found in [14] and then generalised in [15]. Here we study theories with non-analytic choices of free functions characterizing them. These cases have not been much explored in the vectortensor case, and have several interesting features that we point out for the first time. In Section 2 we 
show that such choices of free functions are associated with new symmetries, which can lead to systems propagating less than the expected number of degrees of freedom. The symmetries we identify allow us to single out specific vector-tensor Galileon systems, and we analyse the properties of spherically symmetric and slowly rotating configurations in these systems. In Section 3 we analyse a set-up where the vectortensor action is accompanied by an Einstein-Hilbert contribution, as well as by an arbitrary, unspecified matter Lagrangian, with the only requirement that the latter does not directly couple with the vector field. We prove that stealth solutions exist, whose geometry coincides exactly with the one found in Einstein gravity coupled with the matter fields, but additionally with a non-trivial profile for the vector with specific integration constants. We interpret these results in terms of the properties of the original action, and the number of degrees of freedom that it propagates. We then show that by adding a standard Maxwell kinetic terms for the vector, one finds configurations that are small modifications of ReissnerNordström black holes. We sum-up in Section 4 with a discussion of possible further developments.

\section{System under consideration}

This Section introduces the special class of vector-tensor theories we examine, and find new symmetries which can lead to systems that do not propagate the vector longitudinal mode.

\subsection{Special vector-tensor theories}

The vector-tensor theories dubbed vector Galileons or generalized Proca [10-12] have been first introduced as vector-tensor versions of Galileon and Horndeski actions. Subsequent investigations started to explore their field theoretical [16] and cosmological [17] ramifications.

The vector-tensor Lagrangians we consider are ${ }^{1}$ :

$$
\begin{aligned}
& L_{(2)}=\sqrt{-g} G_{2}, \\
& L_{(3)}=\sqrt{-g} G_{3} \nabla_{\mu} A^{\mu}, \\
& L_{(4)}=\sqrt{-g}\left[G_{4} R+G_{4, X}\left[\left(\nabla_{\mu} A^{\mu}\right)^{2}-\nabla_{\rho} A_{\sigma} \nabla^{\sigma} A^{\rho}\right]\right], \\
& L_{(5)}=\sqrt{-g}\left[G_{5} G_{\mu \nu} \nabla^{\mu} A^{\nu}-\frac{1}{6} G_{5, X}\left[\left(\nabla^{\mu} A_{\mu}\right)^{3}-3 \nabla^{\mu} A_{\mu} \nabla_{\rho} A_{\sigma} \nabla^{\sigma} A^{\rho}+2 \nabla_{\rho} A_{\sigma} \nabla^{\gamma} A^{\rho} \nabla^{\sigma} A_{\gamma}\right]\right],
\end{aligned}
$$

where the $G_{i}$ are functions of $X$ only, and

$$
2 X=-A^{\mu} A_{\mu} .
$$

We assume that $A_{\mu}$ has mass dimension one, as suggested by the standard canonical normalization of Maxwell kinetic term. If the vector is curl-free and has no transverse polarizations, we can write $A_{\mu}=\partial_{\mu} \pi$ for a scalar $\pi$, and we obtain the shift-symmetric scalar-tensor Horndeski theories. Since Lagrangians (1)-(4) explicitly depend on the gauge potential $A_{\mu}$, they break the Abelian $U(1)$ gauge symmetry. Around flat space, they generally propagate five degrees of freedom, which can be decomposed into two tensors, two vector transverse modes, and one scalar (the vector longitudinal polarization). As for scalar Galileons, interactions have been selected such to avoid the propagation of a ghostly Ostrogradsky sixth mode $[11,12]$.

There are special cases though, where new interesting symmetries emerge. Consider the following specific choices of functions $G_{i}$ in eqs (1)-(4):

$$
G_{2}=m^{3} \sqrt{X},
$$

\footnotetext{
${ }^{1}$ We number the Lagrangians from 2 to 5 in analogy with the classification of scalar-tensor Horndeski theories: in the vector-tensor case, however, $L_{(1)}$ is absent. Also, in [18], a higher order vector tensor Lagrangian $L_{(6)}$ was introduced: it does not play a role in our discussion, hence we do not consider it.
} 


$$
\begin{aligned}
G_{3} & =m^{2} \ln X, \\
G_{4} & =m \sqrt{X}, \\
G_{5} & =\ln X,
\end{aligned}
$$

with $m$ a parameter with dimension of a mass. Since these choices of functions $G_{i}$ are non analytic, the system spontaneously breaks Lorentz symmetry, and any consistent vacuum requires $A_{\mu} \neq 0$. This can be a relevant feature when applying these systems to cosmology, or when studying spherically symmetric configurations, as we are going to do in the second part of this work.

When the $G_{i}$ are chosen as in eqs (6)-(9), up to overall constants and total derivatives, the vector tensor Lagrangians (1)-(4) can be re-expressed as

$$
L_{(i)}=\sqrt{-g} m^{5-i} A_{\mu} J_{(i)}^{\mu},
$$

with $i=2, . .5$ and the vectors $J_{(i)}^{\mu}$ given by

$$
\begin{aligned}
& J_{(2)}^{\mu}=-2 X^{-1 / 2} A^{\mu}, \\
& J_{(3)}^{\mu}=-X^{-1}\left(A^{\mu} \nabla_{\alpha} A^{\alpha}-A^{\alpha} \nabla^{\mu} A_{\alpha}\right), \\
& J_{(4)}^{\mu}=X^{-1 / 2} G^{\mu \alpha} A_{\alpha}-\frac{X^{-3 / 2}}{4} \epsilon^{\alpha \beta \sigma \delta} \epsilon_{\alpha}{ }^{\mu \gamma \eta} A_{\beta} \nabla_{\sigma} A_{\gamma} \nabla_{\delta} A_{\eta}, \\
& J_{(5)}^{\mu}=\frac{1}{4 X} \epsilon^{\alpha \beta \sigma \delta} \epsilon^{\nu \rho \gamma \mu}\left[R_{\alpha \beta \rho \nu}-\frac{2}{3 X} \nabla_{\alpha} A_{\rho} \nabla_{\beta} A_{\nu}\right] A_{\delta} \nabla_{\sigma} A_{\gamma} .
\end{aligned}
$$

Expressions (10) for the Lagrangians are convenient since the vector equations of motion (eoms) are simply given by

$$
J_{(i)}^{\mu}=0 \quad \text { (vector equations of motion). }
$$

The quantities $J_{(i)}^{\mu}$ are the vector analogues of the conserved currents in shift symmetric scalar-tensor Horndeski theories, see e.g. [4]. The non analytic choice of functions $G_{i}$ might seem pathological or, at the very least, too specific for exploring phenomenological consequences as properties of black hole solutions. On the other hand, these vector tensor theories have important distinctive features, which further motivate their study. They are candidates for the building blocks of 'massless' set-ups, since can propagate less than five degrees of freedom, even in absence of an Abelian gauge symmetry. We support this statement by means of two arguments, based on symmetry properties. In Section 2.2 we analyse a decoupling limit, where a new global symmetry arises for all the theories above, preventing a scalar mode to acquire a dynamics, and allowing the propagation of at most four degrees of freedom. In Section 2.3, we show that some of the theories above enjoy a new symmetry even outside the decoupling limit, which can forbid the propagation of the vector longitudinal polarization around flat space.

\subsection{Decoupling limit and associated symmetry}

We identify a regime where scalar, vector, and tensor modes are decoupled, and the self-interactions of the longitudinal vector component can be isolated and clearly identified. Such decoupling limit allows us to make manifest a new symmetry characterizing the special Lagrangians that we consider, which prevents the propagation of the scalar mode associated with the longitudinal vector degree of freedom. The study of similar decoupling limits have been essential in the past to identify the dynamics of degrees of freedom in modified gravity theories, such as dRGT massive gravity (see e.g. [19,20] for reviews). In [11], theories of vector Galileons were constructed by demanding that a decoupling limit exists, where the action for 
the scalar longitudinal vector mode obeys a Galileon symmetry. We now discuss a regime which leads to a different symmetry for the system we focus on.

The reference action that we consider is

$$
S=\int d^{4} x\left[\sqrt{-g}\left(M_{P l}^{2} R-\frac{1}{4} F_{\mu \nu}^{2}\right)+\sum_{i=2}^{5} \lambda_{i} L_{(i)}\right],
$$

where the Lagrangian densities $L_{(i)}$ are given in eqs (10) (it is more convenient to work with the Lagrangians as expressed in terms of currents).

First of all, we restore an Abelian gauge symmetry in the system by introducing a Stückelberg field. We make the following substitution whenever we meet a vector potential in our action:

$$
A_{\mu} \rightarrow A_{\mu}-\frac{1}{m} \partial_{\mu} \pi,
$$

with $\pi$ a scalar Stückelberg field of mass dimension one, and for convenience $m$ is the same parameter appearing in the currents (11). After applying the Stückelberg trick, action (16) is invariant under the Abelian gauge symmetry

$$
A_{\mu} \rightarrow A_{\mu}+\frac{1}{m} \partial_{\mu} \xi(x), \quad \pi \rightarrow \pi+\xi(x),
$$

for an arbitrary function $\xi(x)$. We now perturb the metric around Minkowski space:

$$
g_{\mu \nu}=\eta_{\mu \nu}+\frac{1}{M_{P l}} h_{\mu \nu},
$$

where $h_{\mu \nu}$ is the canonically normalised metric fluctuation.

Decoupling proceeds in two steps. First, we decouple gravity sending $M_{P l} \rightarrow \infty$ : all the dependence of the metric fluctuations in the vector part of the action disappears. Then, we decouple the vector transverse modes from the vector longitudinal scalar mode. We do so by considering the simultaneous limits

$$
\begin{aligned}
m & \rightarrow 0, \\
\lambda_{i} m^{4-i} & =\beta_{i} \Lambda^{4-i} \quad \text { remains finite }
\end{aligned}
$$

where $i=2, \ldots 5$ is the index labelling the Lagrangians (10), $\Lambda$ a new energy scale, and $\beta_{i}$ some new finite dimensionless parameters. Notice that, when taking the limit $m \rightarrow 0$, the constants $\lambda_{i}$ must go to infinity (or to zero, depending on the value of $i$ ) in order to keep $\beta_{i}$ finite.

We obtain the following decoupled action around Minkowski space:

$$
S^{d e c}=\int d^{4} x\left[\mathcal{L}_{k i n}\left(h_{\mu \nu}\right)-\frac{1}{4} F_{\mu \nu}^{2}+\sum_{i=2}^{5} \beta_{i} L_{(i)}^{s c a l}\right],
$$

where $\mathcal{L}_{\text {kin }}\left(h_{\mu \nu}\right)$ is the standard quadratic kinetic term for spin two tensor modes. Notice that the three sectors - tensor, vector, scalar - are decoupled as desired. $L_{(i)}^{\text {scal }}$ are the four scalar Lagrangians introduced in [21]:

$$
\begin{aligned}
L_{(2)}^{\text {scal }} & =\Lambda^{2} \sqrt{X_{s}}, \\
L_{(3)}^{\text {scal }} & =\Lambda\left([\Pi]-\frac{1}{X_{s}}[\Phi]\right),
\end{aligned}
$$




$$
\begin{aligned}
L_{(4)}^{\text {scal }} & =\frac{1}{\sqrt{X_{s}}}\left([\Pi]^{2}-\left[\Pi^{2}\right]+\frac{2}{X_{s}}\left(\left[\Phi^{2}\right]-[\Phi][\Pi]\right)\right), \\
L_{(5)}^{\text {scal }} & =\frac{1}{\Lambda X_{s}}\left([\Pi]^{3}+2\left[\Pi^{3}\right]-3\left[\Pi^{2}\right][\Pi]+\frac{3}{X_{s}}\left(2[\Pi]\left[\Phi^{2}\right]-2\left[\Phi^{3}\right]-[\Phi][\Pi]^{2}+[\Phi]\left[\Pi^{2}\right]\right)\right) .
\end{aligned}
$$

where $X_{s}=-\left(\partial_{\mu} \pi \partial^{\mu} \pi\right) / 2, \Pi_{\mu \nu}=\nabla_{\mu} \nabla_{\nu} \pi,\left[\Pi^{n}\right]=\operatorname{Tr} \Pi^{n}$, and $\left[\Phi^{n}\right]=\partial \pi \cdot \Pi^{n} \cdot \partial \pi$. Besides a constant shift symmetry, such Lagrangian densities are invariant - up to total derivatives - under the scalar symmetry

$$
\delta \pi=\pi \omega^{\mu} \partial_{\mu} \pi,
$$

with $\omega^{\mu}$ an arbitrary, constant four-vector. This fact has been shown in [21], to which we refer the reader for further details. Symmetry (27) is more manifest when embedding the system in a higher dimensional brane-world setting. It is inherited by a bulk higher dimensional rotational symmetry, spontaneously broken by the presence of a brane, with $\pi$ playing the role of Goldstone boson (see also [22] for the original papers developing these techniques).

\section{No propagating scalar mode}

This symmetry is very constraining: in fact, it does not even allow for the propagation of standard scalar excitations. In our decoupling limit, we consider as reference metric Minkowski space-time, and a vanishing profile for the vector transverse modes. The scalar equation of motion, and the non-analytic structure of Lagrangians (23)-(26), require a time-like non trivial profile for the scalar field, such that $X_{s}>0$. The scalar equation of motion associated with the decoupled action (22) contains second derivatives of the scalar field, and any background configuration linear in coordinates is a solution: we denote such background configuration

$$
\bar{\pi}(x)=c_{\mu} x^{\mu},
$$

for some arbitrary time-like vector $c_{\mu}$. We consider the case of constant time-like vector $c_{\mu}$, and choose a frame where $c_{\mu}=\left(c_{0}, 0,0,0\right)$, with $c_{0}$ constant, so to preserve spatial isotropy. This scalar background spontaneously breaks Lorentz symmetry, and furthermore reduces the scalar symmetry (27) to

$$
\delta \pi=\pi \omega^{a} \partial_{a} \pi
$$

for an arbitrary three spatial vector $\omega^{a}$ (with $a=1,2,3$ ). This residual symmetry tells us much about the (absence of) dynamics of scalar excitations. We denote perturbations around the background scalar profile as $\hat{\pi}$ :

$$
\pi(x)=\bar{\pi}(t)+\hat{\pi} .
$$

The action of quadratic fluctuations for $\hat{\pi}$ around the scalar background satisfies a linearised version of symmetry (29), i.e.

$$
\delta \hat{\pi}=\bar{\pi}(t) \omega^{a} \partial_{a} \hat{\pi} .
$$

But such residual symmetry prevents the existence of a term quadratic in time derivatives in the quadratic action for $\hat{\pi}$. Such term would violate the residual symmetry (31), since the former is not invariant under the latter:

$$
\begin{aligned}
\delta \int d^{4} x\left(\frac{1}{2} \dot{\hat{\pi}}^{2}\right) & =\int d^{4} x \dot{\hat{\pi}} \partial_{t} \delta \hat{\pi} \\
& =\int d^{4} x \dot{\hat{\pi}}\left(c_{0} \omega^{a} \partial_{a} \hat{\pi}+c_{0} t \omega^{a} \partial_{a} \dot{\hat{\pi}}\right) \\
& =c_{0} \int d^{4} x \dot{\hat{\pi}} \omega^{a} \partial_{a} \hat{\pi} \neq 0 .
\end{aligned}
$$


In the last step we integrated by parts, and removed a total derivative. This fact shows that, around flat space and a time-like scalar background, scalar excitations are non-dynamical since a symmetry prevents them to acquire standard kinetic terms. We identified a decoupling limit where a new symmetry arises, and the action (22) propagates only four degrees of freedom, two tensors and two vectors.

\subsection{A symmetry outside the decoupling limit}

It is natural to ask whether the results of Section 2.2 remain valid also outside a decoupling limit, in particular whether the theory described by Lagrangians (10) propagates four or less degrees of freedom. The analysis in this case is made more subtle by the fact that the theory spontaneously breaks Lorentz symmetry, due to the presence of non-analytic functions in the formulation of the action. A proper Hamiltonian analysis would require the classification of primary and secondary constraints, but this goes beyond the scope of this work. For studies of related problems in the context of scalar-tensor theories, see e.g. [23,24].

Nevertheless, again using symmetry arguments, we are able to provide indications that our systems propagate less than the expected five $d o f s$, at least for some of the Lagrangians of eqs (10), and around certain backgrounds. We impose a discrete parity symmetry to the vector-tensor theories we consider

$$
A_{\mu} \rightarrow-A_{\mu},
$$

which singles out Lagrangians $L_{2}$ and $L_{4}$ from the system of eqs (10):

$$
\begin{aligned}
& L_{2}=m^{3} \sqrt{-g} \sqrt{X}, \\
& L_{4}=m \sqrt{-g}\left[\sqrt{X} R+\frac{1}{2 \sqrt{X}}\left[\left(\nabla_{\mu} A^{\mu}\right)^{2}-\nabla_{\rho} A_{\sigma} \nabla^{\sigma} A^{\rho}\right]\right] .
\end{aligned}
$$

These Lagrangians are particularly important for the analysis of spherically symmetric configurations describing compact objects, as we shall discuss in the next section.

Lagrangian $L_{(2)}$ of eq (34) and a modification of $L_{(4)}$ given by

$$
\tilde{L}_{(4)}=m \sqrt{-g}\left[\sqrt{X} R+\frac{1}{2 \sqrt{X}}\left[\left(\nabla_{\mu} A^{\mu}\right)^{2}-\nabla_{\rho} A_{\sigma} \nabla^{\sigma} A^{\rho}-\frac{1}{4} F_{\mu \nu}^{2}\right]\right]
$$

are independently invariant under a new gauge symmetry acting on the metric only,

$$
g_{\mu \nu} \rightarrow g_{\mu \nu}+\partial_{\mu} \xi A_{\nu}+A_{\mu} \partial_{\nu} \xi
$$

for arbitrary scalar function $\xi$. We checked this statement by brute force using the Mathematica package xAct [25]. In Appendix A, we explain the heuristic method we used to deduce the existence of this symmetry: it arises from a certain limit of a disformal transformation acting on theories equipped by standard Abelian gauge invariance.

It would be interesting to derive in full generality the consequences of this new symmetry, in particular for what respect the total number of degrees of freedom which it allows to propagate ${ }^{2}$. Here we focus our attention to the dynamics of fluctuations around flat space: we support the findings of Section 2.2 but this time taking into full account the coupling between gravity and the vector longitudinal mode.

We 'switch off' the vector transverse modes, and only focus on the vector longitudinal component, which is a scalar whose dynamics we wish to study:

$$
A_{\mu}=\partial_{\mu} \pi .
$$

\footnotetext{
${ }^{2}$ In particular, it would be interesting to understand whether this symmetry - in the the scalar-tensor case where $A_{\mu}=\partial_{\mu} \phi$ - provides a deeper reason for the fact that the scalar-tensor versions of $L_{(2),(4)}$ do not propagate tensor modes, see [24].
} 
In this case, Lagrangian $L_{(2)}$ reduces to the cuscuton model, which has been shown not to propagate scalar degrees of freedom [26]: hence we do not consider it here any further. The two Lagrangians $L_{(4)}$ and $\tilde{L}_{(4)}$ of eqs (35) and (36) coincide in this scalar-only limit, and give the scalar Lagrangian

$$
L_{(4)}^{s}=m \sqrt{-g}\left[\sqrt{X_{s}} R+\frac{1}{2 \sqrt{X_{s}}}\left[\left(\nabla_{\mu} \partial^{\mu} \pi\right)^{2}-\nabla_{\rho} \partial_{\sigma} \pi \nabla^{\sigma} \partial^{\rho} \pi\right]\right],
$$

which describes interactions of the vector longitudinal mode with gravity and with itself. This scalar Lagrangian is invariant under a scalar-tensor limit of symmetry (37):

$$
g_{\mu \nu} \rightarrow g_{\mu \nu}+\partial_{\mu} \xi \partial_{\nu} \pi+\partial_{\mu} \pi \partial_{\nu} \xi
$$

for arbitrary $\xi$.

We now add an Einstein-Hilbert term to the system and analyse the action

$$
S=\int d^{4} x \sqrt{-g}\left[M_{P l}^{2} R+\lambda_{4} L_{(4)}^{s}\right],
$$

describing gravity coupled with the vector longitudinal mode. We show that symmetries available standard diffeomorphisms, as well as the new symmetry (40) - prevent the propagation of the scalar excitation around flat space. Notice that the analysis is made more subtle by the fact that, while $L_{(4)}^{s}$ respects the new symmetry (40), the Einstein-Hilbert term is in general not invariant under this symmetry (although it might be in specific cases).

Flat space $g_{\mu \nu}=\eta_{\mu \nu}$ is a background solution for the equations of motion associated with the action (41), for any constant time-like vector background

$$
\bar{\pi} \equiv \bar{c}_{\mu} x^{\mu} .
$$

We study the dynamics of fluctuations around this background. Perturbations are denoted as

$$
\begin{aligned}
g_{\mu \nu} & =\eta_{\mu \nu}+\hat{h}_{\mu \nu}, \\
\pi & =\bar{\pi}+\hat{\pi},
\end{aligned}
$$

with $\hat{h}_{\mu \nu}$ metric fluctuations, and $\hat{\pi}$ the scalar fluctuations.

Local infinitesimal diffeomorphisms act on metric and scalar fluctuations as

$$
\begin{aligned}
\hat{h}_{\mu \nu} & \rightarrow \hat{h}_{\mu \nu}+\partial_{\mu} \alpha_{\nu}+\partial_{\nu} \alpha_{\mu}, \\
\hat{\pi} & \rightarrow \hat{\pi}+\bar{c}_{\rho} \alpha^{\rho},
\end{aligned}
$$

for an arbitrary function $\alpha^{\mu}$ of small size, with $\bar{c}_{\rho}$ the vector controlling the scalar background (42). A non-vanishing background for $\bar{\pi}$ spontaneously breaks diffeomorphisms (introducing the last contribution to eq (46)). Symmetry (40), distinctive of the $L_{(4)}^{s}$ contribution to action (41), gets also spontaneously broken by the scalar vev. It acts on the metric fluctuations as

$$
\hat{h}_{\mu \nu} \rightarrow \hat{h}_{\mu \nu}+\bar{c}_{\mu} \partial_{\nu} \xi+\bar{c}_{\nu} \partial_{\mu} \xi
$$

for an arbitrary quantity $\xi$ of small size. Notice that symmetry (47) is equivalent to a linearised diffeomorphism acting on the metric only, hence in this case the Einstein-Hilbert term is invariant under this transformation as well.

To analyse the dynamics of scalar fluctuations, we select a gauge and choose a convenient profile for the diffeomorphism parameter $\alpha^{\mu}$ :

$$
\alpha^{\mu}=-\left(\frac{\bar{c}^{\mu}}{\bar{c}^{\rho} \bar{c}_{\rho}}\right) \hat{\pi} .
$$


Such unitary gauge moves the fluctuation $\hat{\pi}$ from the scalar to the metric fluctuations, that gets a contribution depending on derivatives of $\hat{\pi}$ (see eq (45)):

$$
\hat{h}_{\mu \nu}-\frac{\bar{c}_{\mu} \partial_{\nu} \hat{\pi}}{\bar{c}^{\rho} \bar{c}_{\rho}}-\frac{\bar{c}_{\nu} \partial_{\mu} \hat{\pi}}{\bar{c}^{\rho} \bar{c}_{\rho}} .
$$

In principle, $\hat{\pi}$ might acquire dynamics when expanding $L_{(4)}^{s}$ quadratically in fluctuations. However, we can still exploit the symmetry (47), to show that $\hat{\pi}$ is a non-dynamical gauge mode also for $L_{(4)}^{s}$. Making the choice

$$
\xi^{\mu}=\frac{\partial^{\mu} \hat{\pi}}{\bar{c}^{\rho} \bar{c}_{\rho}}
$$

in eq (47) we can remove the $\pi$ contribution from (49). Hence, around flat space, the scalar mode does not propagate.

It would be interesting to further exploit consequences of symmetry (37) to study in full detail the dynamics of fluctuations also around more general background configurations, and including transverse vector modes. As the brief analysis above shows, the number of propagating degrees of freedom should be controlled on the symmetries available around a given configuration. We leave this issue for the future. In the next Section we are going to examine the physics of spherically symmetric and slowly rotating solutions to the equations of motion associated with specific vector-tensor actions.

\section{$3 \quad$ Spherically symmetric and slowly rotating solutions}

In the previous Section, we discussed candidates of vector-tensor theories that can propagate less than the expected five degrees of freedom, even in absence of an Abelian gauge symmetry. Vector-tensor theories of gravity that do not propagate the vector longitudinal mode can be interesting for phenomenology, since they automatically avoid long range fifth forces associated with light scalar fields. In this Section, we discuss ramifications of these theories for configurations that can describe compact objects. We focus on a subset of these actions which obey a further parity symmetry $A_{\mu} \rightarrow-A_{\mu}$ : the Lagrangians $L_{(2),(4)}$ given by eqs (34), (35),

$$
\begin{aligned}
& L_{(2)}=m^{3} \sqrt{-g} \sqrt{X}, \\
& L_{(4)}=m \sqrt{-g}\left[\sqrt{X} R+\frac{1}{2 \sqrt{X}}\left[\left(\nabla_{\mu} A^{\mu}\right)^{2}-\nabla_{\rho} A_{\sigma} \nabla^{\sigma} A^{\rho}\right]\right] .
\end{aligned}
$$

We examine spherically symmetric and slowly rotating configurations associated with such Lagrangians.

In Section 3.1 we consider an action which includes the Einstein-Hilbert contribution, plus Lagrangians $L_{(2)}$ and $L_{(4)}$ of eqs (51), (52). We prove a theorem on the existence of stealth configurations in the presence of arbitrary additional matter besides the vector-tensor Lagrangians we consider. The resulting regular solutions have the same geometry as in Einstein gravity coupled with matter, and a non-trivial vector profile characterized by independent vector charges. We also consider physical consequences of our findings in terms of the degrees of freedom the system can propagate, making further connections with the results of Section 2.3. These results can be important to describe configurations describing black holes or neutron stars in modified theories of gravity including more realistic astrophysical matter forming or surrounding the object.

In Section 3.2 we add to the action the standard Maxwell kinetic term for the vector fields in the action, and we show that spherically symmetric configurations correspond to a Reissner-Nordström configuration, plus small subleading corrections at large radial distances. We discuss how the geometry depends on features of Lagrangians $L_{(2)}$ and $L_{(4)}$. 


\subsection{Stealth spherically symmetric and slowly rotating configurations}

Consider the action

$$
S=\int d^{4} x \sqrt{-g}\left[M_{P l}^{2} R+\lambda_{2} L_{(2)}+\lambda_{4} L_{(4)}+\mathcal{L}_{\text {matter }}\right]
$$

with $L_{(2),(4)}$ the vector-tensor Lagrangian densitites of eqs (51), (52). $\mathcal{L}_{\text {matter }}$ describes an arbitrary matter Lagrangian, with the only condition that matter does not directly couple with the vector field $A_{\mu}$ (but only indirectly through gravity). We prove that the system admits spherically symmetric solutions - as well as solutions in slow rotation - which coincide exactly with the ones of Einstein gravity coupled with $\mathcal{L}_{\text {matter }}$ (i.e. with the solutions obtained without the $L_{(2),(4)}$ contribution to the previous action). These are examples of stealth spherically symmetric configurations with a non-trivial profile for the vector field.

\section{The general proof}

The metric Ansatz we adopt for a general spherically symmetric configuration is

$$
d s^{2}=-F(r) d t^{2}+2 D(r) d t d r+H(r) d r^{2}+r^{2} d \Omega^{2}
$$

We choose an Ansatz for the gauge field profile preserving the spherical symmetry, and with only the time-component turned on

$$
A_{\mu}=\left(A_{0}(r), 0,0,0\right) .
$$

When considering spherically symmetric configurations and the metric Ansatz (54), the radial profile of the metric component $D(r)$ or of $H(r)$ can be chosen arbitrarily by means of a coordinate redefinition. Then the remaining metric components are uniquely determined by the equations of motion. Indeed, a shift of time coordinate

$$
d t \rightarrow d t+\frac{G(r)}{F(r)} d r
$$

for some arbitrary function $G(r)$ leads to the following metric and vector field profiles:

$$
\begin{gathered}
d \tilde{s}^{2}=-F(r) d t^{2}+2(D(r)-G(r)) d t d r+\left(H(r)+\frac{G^{2}(r)}{F(r)}\right) d r^{2}+r^{2} d \Omega^{2} \\
\tilde{A}_{\mu}=\left(A_{0}(r), \frac{G(r) A_{0}(r)}{F(r)}, 0,0\right) .
\end{gathered}
$$

Usually such coordinate freedom is used to remove the off-diagonal component, making the specific choice $G=D$ in eq (62). On the other hand, in what comes next we need to have gauge freedom to choose a profile for $H(r)$, hence we maintain the general Ansatz (54) including the off-diagonal component.

With the metric and field Ansatz (54), (55), we proceed to study the equations of motion. There are four equations to satisfy, associated with the four quantities $A_{0}(r), F(r), D(r), H(r)$.

Plugging the Ansatz in eq (53), we find that $A_{0}(r)$ appears only linearly in the action, and multiplies a combination depending only on $H(r)$. This property is distinctive of our choice for $G_{2,4}$ in eqs (6) and (8). The action results

$$
\begin{aligned}
S= & 4 \pi m \int d r A_{0}(r) H^{-3 / 2}(r)\left[-\sqrt{2} \lambda_{4} H(r)+\left(m^{2} r^{2} \lambda_{2}+\sqrt{2} \lambda_{4}\right) H^{2}(r)+\sqrt{2} \lambda_{4} r H^{\prime}(r)\right] \\
& + \text { parts that do not depend on } A_{0},
\end{aligned}
$$


with the prime denoting a derivative along the radial coordinate. Hence the vector equation of motion for the component $A_{0}(r)$ is special, since it provides a constraint condition on the geometry:

$$
0=-\sqrt{2} \lambda_{4} H+\left(m^{2} r^{2} \lambda_{2}+\sqrt{2} \lambda_{4}\right) H^{2}+\sqrt{2} \lambda_{4} r H^{\prime},
$$

with solution

$$
H(r)=\left(1-\frac{2 \mu}{M_{P l}^{2} r}+\frac{m^{2} \lambda_{2} r^{2}}{6 \lambda_{4}}\right)^{-1},
$$

where $\mu$ is an arbitrary integration constant with dimension of a mass. Recall that, as discussed around (62), the metric function $H(r)$ is a 'pure gauge' and its profile can be modified by changing coordinates. But for the moment we work with the solution (61) for the vector field equation, and proceed to characterise the remaining unknown quantities, determined by the equations of motion associated with the metric components.

The equations of motion for the metric components $F(r), D(r)$ are insensitive to the vector component $A_{0}(r)$ (since the dependence of the action on $A_{0}(r)$ is limited to the first line of eq (59)), hence they have the very same solutions as in Einstein gravity coupled with matter Lagrangian $\mathcal{L}_{\text {matter }}$ (in other words, they do not realise the presence of $L_{(2),(4)}$ in eq (53)).

Finally, the equation of motion relative to $H(r)$ depends explicitly on the vector profile, and can be used for determining $A_{0}(r)$. The vector field profile is non-trivial, and is controlled by the geometry (without affecting it). In general, we expect that the vector profile depends on two independent integration constants (besides the ones that control the geometry): one is the integration constant $\mu$ entering in the solution of the eom for $A_{0}(r)$ in eq (61). The other an integration constant associated with the eom for $H(r)$ (we will expand on this statement in a specific example next). After having determined the solution, if one wishes, one can make a coordinate transformation as in eq (62), setting the metric in a diagonal form choosing $G(r)=D(r)$, and turning on a radial component for the vector profile. From the arguments above, the geometry is the one of Einstein gravity coupled with $\mathcal{L}_{\text {matter }}$.

The very same general proof we have developed can be applied to describe configurations in slowrotation, described by the line element [27]

$$
d s^{2}=-F(r) d t^{2}+2 D(r) d t d r+H(r) d r^{2}+r^{2} d \Omega^{2}+2 a r^{2} \sin ^{2} \theta W(r) d \phi d t,
$$

and

$$
A_{\mu}=\left(A_{0}(r), 0,0,0\right),
$$

with $a$ the rotation parameter. The corresponding system of equations can be studied in an expansion at first order in $a$, leading to the very same conclusions we derived above. When $\mathcal{L}_{m}=0$, the function $W(r)$ acquires a profile $W \sim 1 / r^{3}$, the same as in the slow rotation limit of the Kerr configuration in Einstein GR. In presence of matter, the eom for $W(r)$ can be sourced by it.

After this general proof, we now discuss the case with cosmological constant as an example to concretely explain the structure of the solution, and to show the existence of two integration constants associated with the vector profile.

\section{A concrete example: space-time in the presence of a cosmological constant}

It is well known (see e.g. the papers [28], and [9] for a general review) that adding a cosmological constant to generic Horndeski scalar-tensor systems lead to spherically symmetric black hole solutions which are quite different from their counterparts in Einstein gravity i.e. (A)dS-Schwarzschild black holes. On the other hand, for the specific choice of vector-tensor action $(53)$ with $\mathcal{L}_{\text {matter }}=-6 \Lambda$, our arguments 
suggest that there is a solution to the field equations whose geometry coincides with the one of General Relativity.

We can prove this fact directly, proceeding as discussed above. Adopting a spherically symmetric metric and vector field Ansatz as in eqs (54), (55) with an off-diagonal component for the metric, it is straightforward to find the explicit, unique solution:

$$
\begin{aligned}
H(r) & =\left(1-\frac{2 \mu}{M_{P l}^{2} r}+\frac{m^{2} \lambda_{2} r^{2}}{6 \lambda_{4}}\right)^{-1} \\
F(r) & =1-\frac{2 M}{M_{P l}^{2} r}-\frac{\Lambda r^{2}}{M_{P l}^{2}} \\
D^{2}(r) & =\frac{6 \lambda_{4} \Lambda r^{3}+\lambda_{2} m^{2} M_{P l}^{2} r^{3}-12 \lambda_{4}(\mu-M)}{\lambda_{2} m^{2} M_{P l}^{2} r^{3}-12 \lambda_{4} \mu+6 \lambda_{4} r}, \\
A_{0}(r) & =Q\left(\lambda_{4}-\frac{2 \lambda_{4} \mu}{M_{P l}^{2} r}+\frac{\lambda_{2} m^{2} r^{2}}{6}\right)^{1 / 2} .
\end{aligned}
$$

This configuration depends on three integration constants: the parameter $\mu$ (with dimension of mass), which enters in the profile of $H(r)$, and is associated with the eom for $A_{0}(r)$; the mass parameter $M$, which enters in the profile of $F(r)$, and comes from solving the equations of motion for $D(r), F(r)$; and finally, the parameter $Q$ in the profile for $A_{0}(r)$ (with dimension of mass) associated with the eom for $H(r)$.

By doing a coordinate redefinition as explained around eq (62), the metric can be put in a diagonal form, which makes it more recognizable:

$$
d s^{2}=-\left(1-\frac{2 M}{r}-\frac{\Lambda r^{2}}{M_{P l}^{2}}\right) d t^{2}+\frac{d r^{2}}{\left(1-\frac{2 M}{r}-\frac{\Lambda r^{2}}{M_{P l}^{2}}\right)}+r^{2} d \Omega^{2} .
$$

As expected, this is a stealth (A)dS Schwarzschild black hole configuration, which depends on the integration constant $M$, the mass of the black hole. On the other hand, in this frame, the vector profile is non-trivial, and has two components switchted on:

$$
\begin{aligned}
A_{\mu} & =\left(A_{0}(r), \Pi(r), 0,0\right), \\
A_{0}(r) & =Q\left(\lambda_{4}-\frac{2 \lambda_{4} \mu}{M_{P l}^{2} r}+\frac{\lambda_{2} m^{2} r^{2}}{6}\right)^{1 / 2}, \\
\Pi^{2}(r) & =\frac{m Q^{2}}{M_{P l}^{2} r} \frac{\left[\lambda_{2} m^{2} M_{P l}^{2} r^{3}+6 \lambda_{4}\left(2 M+r^{3} \Lambda-2 \mu\right)\right]^{1 / 2}}{\left(1-\frac{2 M}{M_{P l}^{2} r}+\frac{r^{2} \Lambda}{M_{P l}^{2}}\right)} .
\end{aligned}
$$

The vector field profile contains two integration constants: $Q$, which can be interpreted as the standard vector 'electric' charge associated with the vector component $A_{0}$; and $\mu$, a new parameter that contributes to characterize the vector solution.

\section{Physical consequences}

These results ensure that there exist spherically symmetric and slowly rotating solutions of action (53) which coincide with the ones of Einstein gravity, coupled with matter Lagrangian $\mathcal{L}_{\text {matter }}$. At the same time, the vector field acquires a non-trivial profile, which depends on the geometry and extends to asymptotic infinity and is characterized by specific, independent integration constants. These findings 
are useful for embedding these vector-tensor theories of gravity in more realistic settings, including astrophysical matter forming or surrounding compact, gravitationally bound objects.

It is tempting to relate the findings of this Section to the results of Sections 2.2 and 2.3, which indicate that the theories under consideration propagate less than the expected degrees of freedom: possibly, spherically symmetric configurations coincide with the ones of Einstein gravity, because matter in this case does not excite additional vectorial degrees of freedom, which can backreact on the geometry modifying it. It would be interesting to further pursue these arguments to understand how the conditions of finding stealth configurations in modified gravity theories are associated with the number of propagating modes.

It is also interesting to ask how to probe the non-trivial, asymptotic vector profile associated with the geometry, and the vector 'hairs' associated with the specific vector integration constants. A possibility is to couple the vector to probe matter fields, although then backreaction effects of the vector to the geometry should be taken into account. Alternatively, we can give kinetic terms to the vector in the form of a standard Maxwell action: we discuss this case in what follows.

\subsection{Including Maxwell action: small deviations from Reissner-Nordström}

We now investigate how the results of the previous Section are modified when including vector kinetic terms to the system. We consider the action

$$
S=\int d^{4} x \sqrt{-g}\left[M_{P l}^{2} R-\frac{1}{4} F_{\mu \nu} F^{\mu \nu}+\lambda_{2} L_{(2)}+\lambda_{4} L_{(4)}\right],
$$

with the choices (51) and (52) for $L_{(2),(4)}$, and without loss of generality we set $m=M_{P l}$. We find that spherically symmetric solutions, in absence of additional matter, resemble Reissner-Nordström (RN) configurations in certain regimes, with small deviations induced by the vector-tensor Lagrangians $L_{(2),(4)}$.

The Ansatz for the metric and the gauge field we start with is the same as in (54). When the vector kinetic term is absent, $\lambda_{2}$ drives the vector field profile to diverge asymptotically, although the metric remains asymptotically flat. When the vector kinetic term is included, $\lambda_{2}$ spoils the asymptotic flatness of the metric. Therefore, we start with the simpler case $\lambda_{2}=0$. The metric components $F, H$ and $D$ can be written in terms of $A_{0}$ and one integration constant that we call $M . A_{0}(r)$ satisfies the equation

$$
\frac{2 \sqrt{2} \lambda_{4}\left(A_{0}\left(r A_{0}^{\prime \prime}+2 A_{0}^{\prime}\right)+r\left(A_{0}^{\prime}\right)^{2}\right)}{\sqrt{A_{0}\left(2 r A_{0}^{\prime}+A_{0}\right)^{3}}}-\left(r A_{0}^{\prime \prime}+2 A_{0}^{\prime}\right)=0 .
$$

At large distances, this equation admits a parametric solution in terms of an inverse radius expansion

$$
A_{0}=P+\frac{Q}{r}+\sum_{i=2} \frac{a_{i}}{r^{i}},
$$

where the coefficients $a_{i}$ are determined by $P, Q$ and the parameters of the model. $M$ does not appear in these coefficients. For example, the first few values of $a_{i}$ are

$$
\begin{aligned}
& a_{2}=\frac{Q^{2} \lambda_{4}\left(\sqrt{2} P+4 \lambda_{4}\right)}{P^{3}-8 P \lambda_{4}^{2}}, \\
& a_{3}=\frac{Q^{3} \lambda_{4}\left(\sqrt{2} P^{3}+20 P^{2} \lambda_{4}+56 \sqrt{2} P \lambda_{4}^{2}+96 \lambda_{4}^{3}\right)}{3\left(P^{3}-8 P \lambda_{4}^{2}\right)^{2}}, \\
& a_{4}=\frac{Q^{4} \lambda_{4}\left(3 \sqrt{2} P^{5}+88 P^{4} \lambda_{4}+504 \sqrt{2} P^{3} \lambda_{4}^{2}+2784 P^{2} \lambda_{4}^{3}+3712 \sqrt{2} P \lambda_{4}^{4}+3840 \lambda_{4}^{5}\right)}{12\left(P^{3}-8 P \lambda_{4}^{2}\right)^{3}} .
\end{aligned}
$$



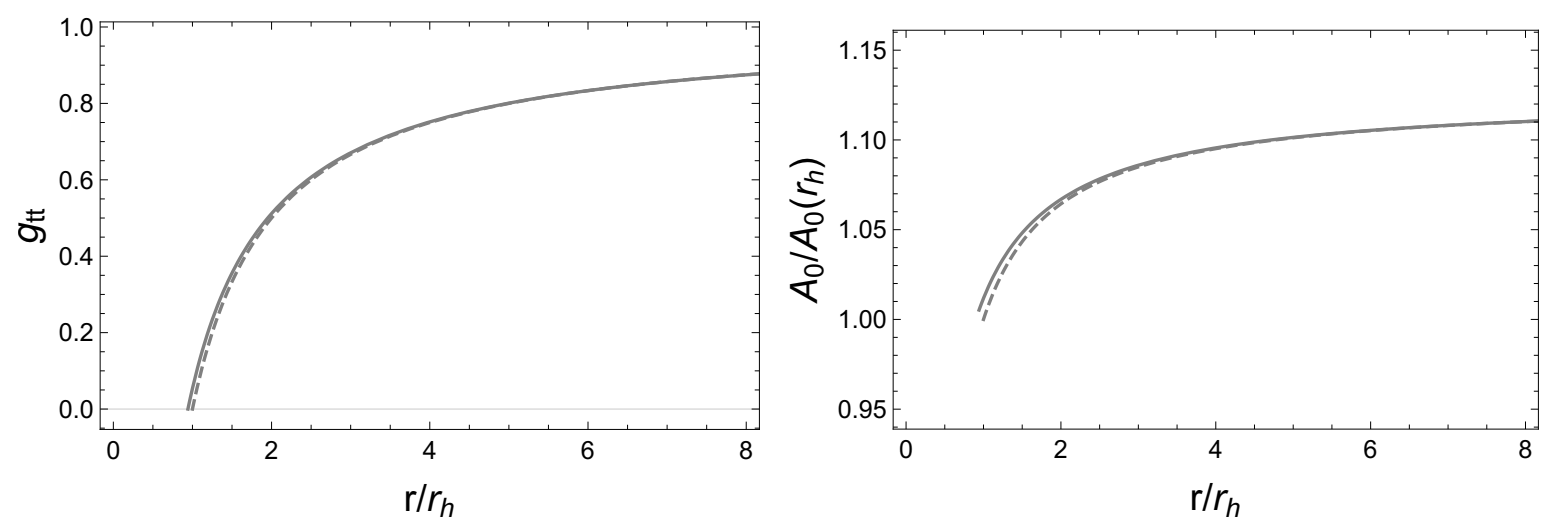

Figure 1: $g^{t t}$ and $A_{0}$ for $\lambda_{2}=0$. The dashed line shows the case without vector kinetic term, where Schwarzschild BH's of radius $r_{h}$ are formed; for both solutions - with and without vector kinetic term the radial coordinate is normalized over this $r_{h}$. When the Maxwell term is on, the horizon position is shifted to smaller values. In both cases, the metric is asymptotically flat and $A_{0}$ asymptotes to a constant value. For these plots, we use $\lambda_{4}=1$, and the asymptotic values $P \approx 5 M_{p}$ and $Q \approx-1600$ and mass $c^{2} M / G=1500 M_{p}$ (in units where $c=G=M_{p}=1$. Restoring the SI values of these constant, this is equivalent to 1 solar mass).

After diagonalising the metric as explained around eq (62), we find

$$
\begin{aligned}
& f=h=1-\frac{2 M}{r}+\frac{Q^{2}}{4 r^{2}}+\frac{Q^{3} \lambda_{4}\left(\sqrt{2} P+4 \lambda_{4}\right)}{2 r^{3}\left(P^{3}-8 P \lambda_{4}^{2}\right)}+\ldots, \\
& \Pi=\sqrt{\frac{2 P(M P+Q)}{r}}+\frac{8 P(2 M P+Q)^{2}-P^{3} Q^{2}-256 M(M P+Q) \lambda_{4}^{2}+8 Q^{2} \lambda_{4}\left(2 \sqrt{2}+P \lambda_{4}\right)}{8 \sqrt{2(M+Q / P)} r^{3 / 2}\left(P^{2}-8 \lambda_{4}^{2}\right)}+\ldots
\end{aligned}
$$

This shows that, at large $r$, the solutions correspond to small deviations from a RN black hole, with a non-trivial profile for the longitudinal mode of the vector field.

To investigate what happens for small $r$ we resort to a numerical analysis. We solve eq. (73) by imposing initial conditions for $A_{0}$ and $A_{0}^{\prime}$ at a large radius $r_{i}$ and evolve inwards. We verify that $r_{i}$ is large enough for the solutions to respect the asymptotic behaviour found analytically, so that we can safely read from the solutions the black hole mass $c^{2} M / G$, and the vector charges $P$ and $Q$. We determine the radius of the black hole horizon $r_{h}$ by identifying the point where $g^{r r}=0$. The results are shown in Fig. 1. The left panel shows the profiles for $g_{t t}=g^{r r}$, and the right panel shows the profiles for $A_{0}$. For comparison, the dashed lines show the case without vector kinetic term, where the metric is exactly Schwarzschild. Only near the horizon the solution deviates from Schwarzschild. Interestingly, the resulting black hole horizon area is smaller than the one of a Schwarzschild or RN black hole with the same mass. This implies that these black holes are more compact than their GR or Einstein-Maxwell counterparts.

Now we turn our attention to the case where both $\lambda_{2}$ and $\lambda_{4}$ are different from zero ${ }^{3}$. Once again, the metric components can be expressed in terms of $A_{0}$, which is determined from the equation

$$
\lambda_{4}\left(2 \lambda_{4}+\lambda_{2} r^{2}\right)\left(2 r A_{0}^{\prime}+A_{0}\right)^{2}-A_{0}\left(2 \lambda_{4}+\lambda_{2} r^{2}\right)\left(2 r A_{0}^{\prime}+A_{0}\right)\left[r \sqrt{\frac{\lambda_{4}\left(2 r A_{0}^{\prime}+A_{0}\right)}{A_{0}\left(2 \lambda_{4}+\lambda_{2} r^{2}\right)}}\left(r A_{0}^{\prime \prime}+2 A_{0}^{\prime}\right)+\lambda_{4}\right]
$$

\footnotetext{
${ }^{3}$ The case $\lambda_{4}=0, \lambda_{2} \neq 0$ does not admit spherically symmetric solutions with a diagonal metric that satisfies $g_{t t}=g^{r r}$.
} 

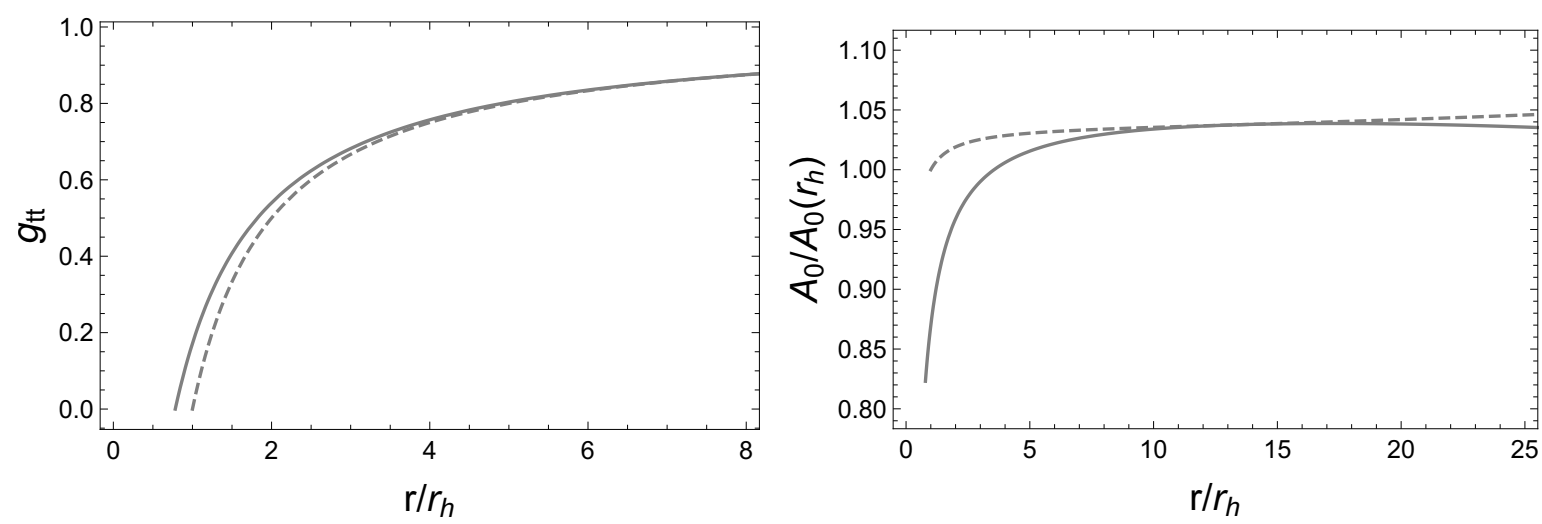

Figure 2: Same as the previous figure but with both $\lambda_{2}$ and $\lambda_{4}$ different from zero. Only when the vector kinetic term is present the asymptotic behaviour of the metric is sensitive to the asymptotic divergence of $A_{0}$. Since we do not have an analytic expression for the asymptotic solution we do not have control over the charges of this solution, therefore we set the same initial conditions as for the case $\lambda_{2}=0$, and choose $\lambda_{4}=1$ and a small value $\lambda_{2}=2 \times 10^{-11}$, such that these initial conditions are approximately valid.

$$
-r \lambda_{4}\left[\left(2 r A_{0}^{\prime}+A_{0}\right)\left[A_{0}^{\prime}\left(2 \lambda_{4}+\lambda_{2} r^{2}\right)+2 A_{0} \lambda_{2} r\right]-A_{0}\left(2 \lambda_{4}+\lambda_{2} r^{2}\right)\left(2 r A_{0}^{\prime \prime}+3 A_{0}^{\prime}\right)\right]=0
$$

It is straightforward to verify that in the limit $\lambda_{2} \rightarrow 0$ this reduces to eq. (73). Since the solutions are not asymptotically flat, analytic expansions are difficult to implement, therefore we show only numerical results. To set initial conditions we assume a small value of $\lambda_{2}$, so that the same initial conditions used for the asymptotically flat solutions with $\lambda_{2}=0$ can be used as an approximation. Fig. 2 shows the metric component $g_{t t}$ and the vector component $A_{0}$. Both the metric and vector field components diverge for large $r$.

Finally, notice that the quantity $X=-\frac{1}{2} A_{\mu} A^{\mu}$ has a non-trivial profile, as shown in Fig. 3 . This is a difference with respect to configurations with no vector kinetic terms. For $\lambda_{2}=0$ we can write down an asymptotic analytic expression for $X$. Up to second order in $1 / r$, it has the form

$$
X=\frac{1}{2} A_{0}\left(A_{0}+2 r A_{0}^{\prime}\right)=\frac{P^{2}}{2}+\frac{P Q^{2}\left(2 \sqrt{2} \lambda_{4}+P\right)}{2 r^{2}\left(8 \lambda_{4}^{2}-P^{2}\right)}+\ldots
$$

For $\lambda_{2} \neq 0$, we can write an expression for $X$ in terms only of $A_{0}$,

$$
X=\frac{A_{0} \lambda_{4}\left(2 r A_{0}^{\prime}+A_{0}\right)}{2 \lambda_{4}+\lambda_{2} r^{2}}
$$

but we cannot approximate its behaviour analytically since we do not know the asymptotic profile of $A_{0}$.

To conclude, in this Section we shown that when including vector kinetic terms, the geometry depends on the details of the vector profile: when $\lambda_{2}=0$, the geometrical solution corresponds to a small deformation from $\mathrm{RN}$ black hole. When $\lambda_{2} \neq 0$, the vector backreaction is more important, and the geometry is no more asymptotically flat. 

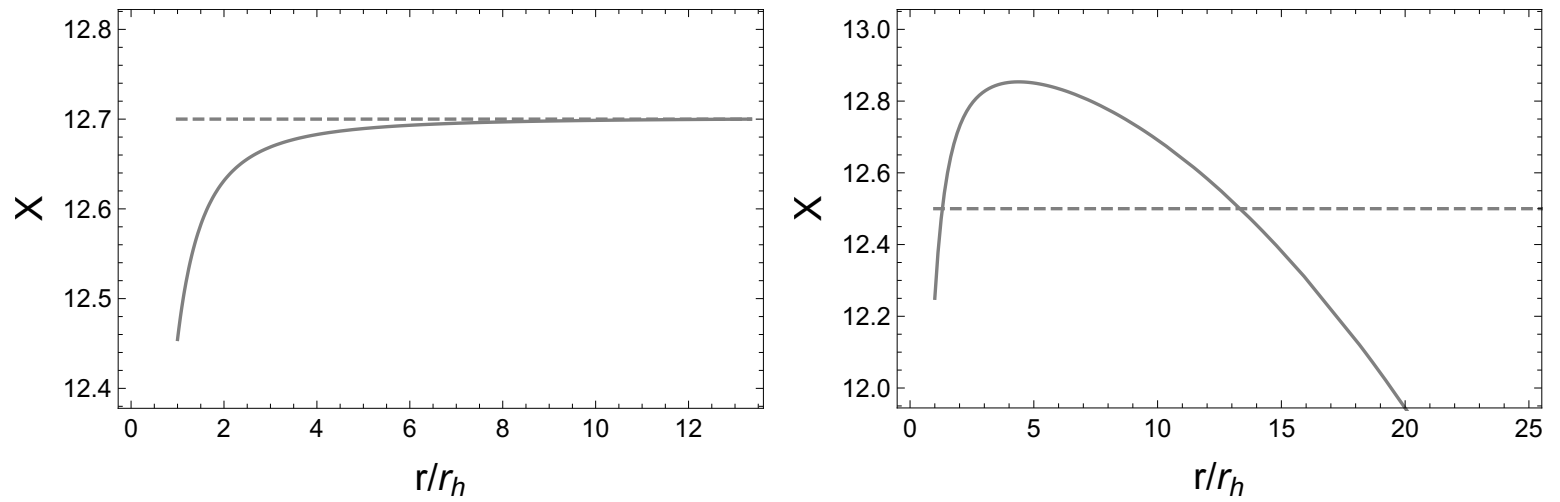

Figure 3: The left panel shows $X$ for $\lambda_{2}=0$ and $\lambda_{4}=1$. The right panel shows $X$ for $\lambda_{2}=2 \times 10^{-11}$ and $\lambda_{4}=1$. In both panels, the dashed line shows the constant value of $X$ obtained when the vector kinetic term is absent.

\section{Discussion}

In this work, we have shown that specific examples of vector-tensor theories of gravity have novel gauge symmetries, which can prevent the propagation of the vector longitudinal degree of freedom, at least around certain backgrounds. Vector-tensor theories of gravity that do not propagate the vector longitudinal mode can be interesting for phenomenology, since they automatically avoid long range fifth forces associated with light scalar fields. We provided an heuristic understanding of these symmetries, as originating from a vector disformal transformation of an Abelian symmetric set-up. We then studied properties of spherically symmetric and slowly rotating configurations in this system. We have shown that a system containing Einstein gravity, vector-tensor Galileons, and an arbitrary matter Lagrangian (with no direct couplings to vectors) admits stealth solutions whose geometry coincides with solutions of Einstein gravity coupled with the matter Lagrangian. Nevertheless the vector field acquires a non-trivial profile, depending on specific integration constants. We commented on a physical interpretation of these results in terms of symmetries of the original action, and how the the vector profile can be probed by directly coupling matter and vectors, as for example in the interior of neutron stars. We also shown that when including standard vector kinetic terms in the form of a Maxwell Lagrangian, solutions for vector-Galileons in vacuum correspond to small modifications of Reissner-Nordstöm configurations.

These results are important to eventually describe gravitationally bound configurations in modified theories of gravity, such as black holes and neutron stars, including realistic matter fields forming or surrounding the object. Future developments can occur in two directions. First, it is important to classify in full generality all consistent vector-tensor theories which propagate only four degrees of freedom, even in absence of Abelian gauge symmetry. These more general theories, interesting for phenomenology, can be equipped by some alternative gauge symmetries, or simply enjoy second class constraints which prevent the propagation of additional modes. The lessons learned in studying these classes of theories can then be used for other contexts, as in scalar-tensor systems or, optimistically, to find explicit examples of partially massless massive gravity models. Second, our findings can be useful for further studying the physics of gravitationally bound compact objects in modified gravity, and for relating their properties to symmetries or features of the systems one examines. The existence of stealth solutions ensures that the starting background configuration coincides with the one of Einstein gravity, but can lead to sizeable differences when considering the dynamics of fluctuations, or when coupling vector and matter fields. We hope to report soon on new results along these directions. 


\section{Acknowledgments}

It is a pleasure to thank Marco Crisostomi, Carlos Núnez, and Ivonne Zavala for suggestions. JC is supported by CONACyt grants 263819 and 179208 .

\section{A Disformal transformation and symmetries}

In this Appendix, we show that the vector-tensor Lagrangians

$$
\begin{aligned}
& L_{2}=m^{3} \sqrt{-g} \sqrt{X} \\
& \tilde{L}_{4}=m \sqrt{-g}\left\{\sqrt{X} R+\frac{1}{2 \sqrt{X}}\left[\left(\nabla_{\mu} A^{\mu}\right)^{2}-\nabla_{\rho} A_{\sigma} \nabla^{\sigma} A^{\rho}-\frac{1}{4} F_{\mu \nu} F^{\mu \nu}\right]\right\},
\end{aligned}
$$

can be obtained from a certain limit of a disformal transformation acting on an Einstein-Hilbert system. This is useful to exhibit a new symmetry that these Lagrangians satisfy.

\section{Details of the disformal transformation}

We examine the following disformal transformation of the metric [29], which involves vector degrees of freedom [30] (but see also [31] for important papers discussing consequences of disformal transformations in scalar-tensor systems):

$$
g_{\mu \nu} \rightarrow \bar{g}_{\mu \nu}=g_{\mu \nu}-\frac{1}{\epsilon^{2} m^{2}} A_{\mu} A_{\nu}
$$

with inverse

$$
\bar{g}^{\mu \nu}=g^{\mu \nu}+\frac{\gamma_{0}^{2}}{m^{2}} A^{\mu} A^{\nu} .
$$

We define

$$
\gamma_{0}^{2}=\frac{m^{2}}{m^{2} \epsilon^{2}+2 X},
$$

and $2 X=-A^{2}$. Here $m$ is a mass scale (for definiteness, the same mass scale appearing in eqs (82), (83)), and $\epsilon$ an arbitrary dimensionless parameter, which we consider as small for the sake of our arguments. We apply the disformal transformation to a Lagrangian made of Einstein-Hilbert, Maxwell, and cosmological constant terms:

$$
\epsilon L=\sqrt{-g}\left[\epsilon M_{P l}^{2} R+\epsilon \Lambda-\frac{1}{4 \epsilon} F_{\mu \nu} F^{\mu \nu}\right] .
$$

We weight parts of the Lagrangian density with powers of the parameter $\epsilon$ before performing the transformation, this will be important in what follows.

The disformal transformation, when applied to the cosmological constant term, gives

$$
\begin{aligned}
\epsilon \Lambda \sqrt{-g} \rightarrow \epsilon \Lambda \sqrt{-\bar{g}} & =\gamma_{0}^{-1} \Lambda \sqrt{-g} \\
& =\frac{\Lambda}{m} \sqrt{2 X} \sqrt{-g}+\mathcal{O}\left(\epsilon^{2}\right) .
\end{aligned}
$$

In the $\epsilon \rightarrow 0$ limit, the resulting Lagrangian after the transformation is $L_{(2)}$, given in eq (82).

While for the Einstein-Hilbert plus Maxwell Lagrangian, we find that the disformed contribution is

$$
\epsilon M_{P l}^{2} \sqrt{-g} R-\frac{\sqrt{-g}}{4 \epsilon} F_{\mu \nu} F^{\mu \nu} \rightarrow \epsilon M_{P l}^{2} \sqrt{-\bar{g}} \bar{R}-\frac{\sqrt{-\bar{g}}}{4 \epsilon} F_{\mu \nu} F_{\rho \sigma} \bar{g}^{\mu \rho} \bar{g}^{\nu \sigma},
$$


with

$$
\begin{gathered}
\sqrt{-\bar{g}}\left[\epsilon M_{P l}^{2} \bar{R}-\frac{1}{4 \epsilon} F_{\mu \nu} F_{\rho \sigma} \bar{g}^{\mu \rho} \bar{g}^{\nu \sigma}\right]=\sqrt{-g} \frac{M_{P l}^{2}}{m}\left\{\gamma_{0}^{-1} R+\gamma_{0}\left[\left(\nabla_{\mu} A^{\mu}\right)^{2}-\nabla_{\mu} A^{\nu} \nabla_{\nu} A^{\mu}-\frac{1}{4} F^{2}\right]\right\} \\
=\sqrt{-g} \frac{M_{P l}^{2}}{m}\left\{\sqrt{2 X} R+\frac{1}{\sqrt{2 X}}\left[\left(\nabla_{\mu} A^{\mu}\right)^{2}-\nabla_{\mu} A^{\nu} \nabla_{\nu} A^{\mu}-\frac{1}{4} F^{2}\right]\right\}+\mathcal{O}\left(\epsilon^{2}\right),
\end{gathered}
$$

plus total derivatives. In the previous expression, $F^{2}=F_{\mu \nu} F^{\mu \nu}$. In the $\epsilon \rightarrow 0$ limit, the resulting Lagrangian is $\tilde{L}_{(4)}$ given in eq (83).

\section{An heuristic derivation of a new symmetry}

Hence the initial Einstein-Maxwell action, equipped with a cosmological constant, is disformally equivalent to a set of vector-tensor theories, labelled by a dimensionless quantity $\epsilon$ which parameterises the disformal transformation. The original action has an Abelian gauge symmetry, while the final system is not Abelian symmetric. Nevertheless, we would expect that some form of memory of the original symmetry remains. Indeed, we are going to show that a new gauge symmetry arises for the disformed theory.

The disformal transformation we are examining is built in terms of the tensor object

$$
g_{\mu \nu} \rightarrow \bar{g}_{\mu \nu}=g_{\mu \nu}-\frac{1}{\epsilon^{2} m^{2}} A_{\mu} A_{\nu} .
$$

The vector does not necessarily transform under disformal transformation. On the other hand, recalling that the original theory is invariant under gauge transformation, being built in terms of $F_{\mu \nu}$, we can disformally map the vector to

$$
A_{\mu} \rightarrow \bar{A}_{\mu}=A_{\mu}+\partial_{\mu} f,
$$

for an arbitrary function $f$ : the result of the disformal transformation is independent from $f$.

These two facts imply that any transformation acting on the fields $g_{\mu \nu}, A_{\mu}$, which leaves invariant expressions (93), (94), is a symmetry of the system, obtained after applying the disformal transformation. An example of symmetry is:

$$
\begin{aligned}
A_{\mu} & \rightarrow A_{\mu}+\epsilon^{2} m^{2} \partial_{\mu} \xi, \\
g_{\mu \nu} & \rightarrow g_{\mu \nu}+\partial_{\mu} \xi A_{\nu}+A_{\mu} \partial_{\nu} \xi+\epsilon^{2} m^{2} \partial_{\mu} \xi \partial_{\nu} \xi,
\end{aligned}
$$

for an arbitrary scalar field $\xi$ (with dimension inverse of the square of a mass). Notice that the vector transforms in eq (95) as an Abelian gauge transformation. The limit $\epsilon \rightarrow 0$ of this transformation is well defined, and consists of a transformation which only acts on the metric:

$$
g_{\mu \nu} \rightarrow g_{\mu \nu}+A_{\nu} \partial_{\mu} \xi+A_{\mu} \partial_{\nu} \xi .
$$

Hence transformation (97) is a symmetry for the Lagrangians of eqs (82), (83). It would be interesting to develop this method, based on disformal transformations, to find new symmetris for more general scalar Horndeski and vector tensor actions starting from specific Abelian symmetric actions.

\section{References}

[1] T. Clifton, P. G. Ferreira, A. Padilla and C. Skordis, Phys. Rept. 513 (2012) 1 doi:10.1016/j.physrep.2012.01.001 [arXiv:1106.2476 [astro-ph.CO]]. 
[2] J. D. Bekenstein, "Black hole hair: 25 - years after," In *Moscow 1996, 2nd International A.D. Sakharov Conference on physics* 216-219 [gr-qc/9605059].

[3] G. W. Horndeski, Int. J. Theor. Phys. 10 (1974) 363. doi:10.1007/BF01807638

[4] L. Hui and A. Nicolis, Phys. Rev. Lett. 110 (2013) 241104 doi:10.1103/PhysRevLett.110.241104 [arXiv:1202.1296 [hep-th]].

[5] T. P. Sotiriou and S. Y. Zhou, Phys. Rev. Lett. 112 (2014) 251102 doi:10.1103/PhysRevLett.112.251102 [arXiv:1312.3622 [gr-qc]].

[6] T. P. Sotiriou and S. Y. Zhou, Phys. Rev. D 90 (2014) 124063 doi:10.1103/PhysRevD.90.124063 [arXiv:1408.1698 [gr-qc]].

[7] C. A. R. Herdeiro and E. Radu, Int. J. Mod. Phys. D 24 (2015) no.09, 1542014 doi:10.1142/S0218271815420146 [arXiv:1504.08209 [gr-qc]].

[8] T. P. Sotiriou, Class. Quant. Grav. 32 (2015) no.21, 214002 doi:10.1088/0264-9381/32/21/214002 [arXiv:1505.00248 [gr-qc]].

[9] M. S. Volkov, arXiv:1601.08230 [gr-qc].

[10] B. M. Gripaios, JHEP 0410, 069 (2004) doi:10.1088/1126-6708/2004/10/069 [hep-th/0408127];

[11] G. Tasinato, JHEP 1404, 067 (2014) doi:10.1007/JHEP04(2014)067 [arXiv:1402.6450 [hep-th]];

[12] L. Heisenberg, JCAP 1405, 015 (2014) doi:10.1088/1475-7516/2014/05/015 [arXiv:1402.7026 [hep-th]].

[13] J. D. Bekenstein, Phys. Rev. D 5 (1972) 1239. doi:10.1103/PhysRevD.5.1239; J. D. Bekenstein, Phys. Rev. D 5 (1972) 2403. doi:10.1103/PhysRevD.5.2403

[14] J. Chagoya, G. Niz and G. Tasinato, Class. Quant. Grav. 33, no. 17, 175007 (2016) doi:10.1088/02649381/33/17/175007 [arXiv:1602.08697 [hep-th]].

[15] M. Minamitsuji, Phys. Rev. D 94 (2016) no.8, 084039 doi:10.1103/PhysRevD.94.084039 [arXiv:1607.06278 [gr-qc]]; A. Cisterna, M. Hassaine, J. Oliva and M. Rinaldi, Phys. Rev. D 94 (2016) no.10, 104039 doi:10.1103/PhysRevD.94.104039 [arXiv:1609.03430 [gr-qc]]; E. Babichev, C. Charmousis and M. Hassaine, JHEP 1705 (2017) 114 doi:10.1007/JHEP05(2017)114 [arXiv:1703.07676 [gr-qc]]; J. Chagoya, G. Niz and G. Tasinato, Class. Quant. Grav. 34 (2017) no.16, 165002 [arXiv:1703.09555 [gr-qc]]; L. Heisenberg, R. Kase, M. Minamitsuji and S. Tsujikawa, arXiv:1705.09662 [gr-qc]; L. Heisenberg, R. Kase, M. Minamitsuji and S. Tsujikawa, arXiv:1706.05115 [gr-qc].

[16] N. Khosravi, Phys. Rev. D 89 (2014) no.12, 124027 doi:10.1103/PhysRevD.89.124027 [arXiv:1404.7503 [hep-th]]; P. Fleury, J. P. Beltran Almeida, C. Pitrou and J. P. Uzan, JCAP 1411 (2014) no.11, 043 doi:10.1088/1475-7516/2014/11/043 [arXiv:1406.6254 [hep-th]]; M. Hull, K. Koyama and G. Tasinato, JHEP 1503 (2015) 154 doi:10.1007/JHEP03(2015)154 [arXiv:1408.6871 [hep-th]]; M. Hull, K. Koyama and G. Tasinato, Phys. Rev. D 93 (2016) no.6, 064012 doi:10.1103/PhysRevD.93.064012 [arXiv:1510.07029 [hep-th]]; F. Charmchi, Z. Haghani, S. Shahidi and L. Shahkarami, Phys. Rev. D 93 (2016) no.12, 124044 doi:10.1103/PhysRevD.93.124044 [arXiv:1511.07034 [hep-th]]; J. Chagoya and G. Tasinato, JHEP 1602 (2016) 063 doi:10.1007/JHEP02(2016)063 [arXiv:1511.07805 [hep-th]]; L. Heisenberg, R. Kase and S. Tsujikawa, Phys. Lett. B 760 (2016) 617 doi:10.1016/j.physletb.2016.07.052 [arXiv:1605.05565 [hep-th]]; A. Amado, Z. Haghani, A. Mohammadi and S. Shahidi, Phys. Lett. B 772 (2017) 141 doi:10.1016/j.physletb.2017.06.040 [arXiv:1612.06938 [hep-th]]. 
[17] G. Tasinato, Class. Quant. Grav. 31 (2014) 225004 doi:10.1088/0264-9381/31/22/225004 [arXiv:1404.4883 [hep-th]]; A. De Felice, L. Heisenberg, R. Kase, S. Tsujikawa, Y. l. Zhang and G. B. Zhao, Phys. Rev. D 93 (2016) no.10, 104016 doi:10.1103/PhysRevD.93.104016 [arXiv:1602.00371 [gr-qc]]; C. M. Nieto and Y. Rodriguez, Mod. Phys. Lett. A 31 (2016) no.21, 1640005 doi:10.1142/S0217732316400058 [arXiv:1602.07197 [gr-qc]]; A. De Felice, L. Heisenberg, R. Kase, S. Mukohyama, S. Tsujikawa and Y. l. Zhang, JCAP 1606 (2016) no.06, 048 doi:10.1088/1475-7516/2016/06/048 [arXiv:1603.05806 [gr-qc]]; A. De Felice, L. Heisenberg, R. Kase, S. Mukohyama, S. Tsujikawa and Y. l. Zhang, Phys. Rev. D 94 (2016) no.4, 044024 doi:10.1103/PhysRevD.94.044024 [arXiv:1605.05066 [gr-qc]]; L. Heisenberg, R. Kase and S. Tsujikawa, JCAP 1611 (2016) no.11, 008 doi:10.1088/1475-7516/2016/11/008 [arXiv:1607.03175 [gr-qc]]; S. Nakamura, R. Kase and S. Tsujikawa, Phys. Rev. D 95 (2017) no.10, 104001 doi:10.1103/PhysRevD.95.104001 [arXiv:1702.08610 [gr-qc]]. A. de Felice, L. Heisenberg and S. Tsujikawa, Phys. Rev. D 95 (2017) no.12, 123540 doi:10.1103/PhysRevD.95.123540 [arXiv:1703.09573 [astro-ph.CO]].

[18] E. Allys, P. Peter and Y. Rodriguez, JCAP 1602 (2016) no.02, 004 doi:10.1088/1475-7516/2016/02/004 [arXiv:1511.03101 [hep-th]]; J. Beltran Jimenez and L. Heisenberg, Phys. Lett. B 757 (2016) 405 doi:10.1016/j.physletb.2016.04.017 [arXiv:1602.03410 [hep-th]].

[19] K. Hinterbichler, Rev. Mod. Phys. 84 (2012) 671 doi:10.1103/RevModPhys.84.671 [arXiv:1105.3735 [hep-th]].

[20] C. de Rham, Living Rev. Rel. 17 (2014) 7 doi:10.12942/lrr-2014-7 [arXiv:1401.4173 [hep-th]].

[21] J. Chagoya and G. Tasinato, JHEP 1702 (2017) 113 doi:10.1007/JHEP02(2017)113 [arXiv:1610.07980 [hepth]].

[22] C. de Rham and A. J. Tolley, JCAP 1005 (2010) 015 doi:10.1088/1475-7516/2010/05/015 [arXiv:1003.5917 [hep-th]]; G. Goon, K. Hinterbichler and M. Trodden, JCAP 1107 (2011) 017 doi:10.1088/14757516/2011/07/017 [arXiv:1103.5745 [hep-th]]; G. Goon, K. Hinterbichler and M. Trodden, Phys. Rev. Lett. 106 (2011) 231102 doi:10.1103/PhysRevLett.106.231102 [arXiv:1103.6029 [hep-th]]; C. Burrage, C. de Rham and L. Heisenberg, JCAP 1105 (2011) 025 doi:10.1088/1475-7516/2011/05/025 [arXiv:1104.0155 [hep-th]].

[23] T. j. Chen, M. Fasiello, E. A. Lim and A. J. Tolley, JCAP 1302 (2013) 042 doi:10.1088/14757516/2013/02/042 [arXiv:1209.0583 [hep-th]]; D. Langlois and K. Noui, JCAP 1602 (2016) no.02, 034 doi:10.1088/1475-7516/2016/02/034 [arXiv:1510.06930 [gr-qc]]; M. Crisostomi, M. Hull, K. Koyama and G. Tasinato, JCAP 1603 (2016) no.03, 038 doi:10.1088/1475-7516/2016/03/038 [arXiv:1601.04658 [hepth]]; M. Crisostomi, K. Koyama and G. Tasinato, JCAP 1604 (2016) no.04, 044 doi:10.1088/14757516/2016/04/044 [arXiv:1602.03119 [hep-th]]; J. Ben Achour, D. Langlois and K. Noui, Phys. Rev. D 93 (2016) no.12, 124005 doi:10.1103/PhysRevD.93.124005 [arXiv:1602.08398 [gr-qc]].

[24] D. Langlois and K. Noui, JCAP 1607 (2016) no.07, 016 doi:10.1088/1475-7516/2016/07/016 [arXiv:1512.06820 [gr-qc]]; C. de Rham and A. Matas, JCAP 1606 (2016) no.06, 041 doi:10.1088/14757516/2016/06/041 [arXiv:1604.08638 [hep-th]]; J. Ben Achour, M. Crisostomi, K. Koyama, D. Langlois, K. Noui and G. Tasinato, JHEP 1612 (2016) 100 doi:10.1007/JHEP12(2016)100 [arXiv:1608.08135 [hepth]].

[25] J. M. Martín-Garcia, http://www.xact.es.

[26] N. Afshordi, D. J. H. Chung and G. Geshnizjani, Phys. Rev. D $75 \quad(2007) \quad 083513$ doi:10.1103/PhysRevD.75.083513 [hep-th/0609150]; N. Afshordi, D. J. H. Chung, M. Doran and G. Geshnizjani, Phys. Rev. D 75 (2007) 123509 doi:10.1103/PhysRevD.75.123509 [astro-ph/0702002].

[27] S. M. Carroll, San Francisco, USA: Addison-Wesley (2004) 513 p

[28] M. Rinaldi, Phys. Rev. D 86 (2012) 084048 doi:10.1103/PhysRevD.86.084048 [arXiv:1208.0103 [gr-qc]]; M. Minamitsuji, Phys. Rev. D 89 (2014) 064017 doi:10.1103/PhysRevD.89.064017 [arXiv:1312.3759 [gr-qc]]; A. Anabalon, A. Cisterna and J. Oliva, Phys. Rev. D 89 (2014) 084050 doi:10.1103/PhysRevD.89.084050 [arXiv:1312.3597 [gr-qc]]. 
[29] J. D. Bekenstein, Phys. Rev. D 48 (1993) 3641 doi:10.1103/PhysRevD.48.3641 [gr-qc/9211017].

[30] R. Kimura, A. Naruko and D. Yoshida, JCAP 1701 (2017) no.01, 002 doi:10.1088/1475-7516/2017/01/002 [arXiv:1608.07066 [gr-qc]].

[31] D. Bettoni and S. Liberati, Phys. Rev. D 88 (2013) 084020 doi:10.1103/PhysRevD.88.084020 [arXiv:1306.6724 [gr-qc]]; M. Zumalacrregui and J. Garca-Bellido, Phys. Rev. D 89 (2014) 064046 doi:10.1103/PhysRevD.89.064046 [arXiv:1308.4685 [gr-qc]]. 